\title{
Arbor
}

\section{Los desafíos al sistema de protección social derivados de la postmodernización de la familia *}

Gerardo Meil Landwerlin *

Arbor CLXXIV, 685 (Enero 2003), 163-193 pp.

Históricamente los sistemas de protección social se han desarrollado para garantizar la subsistencia cuando las capacidades individuales y la solidaridad de la unidad familiar en la que se integran los individuos no podían hacer frente a las necesidades de la vida. Inicialmente asumidas por instituciones de carácter religioso y/o gremial, el desarrollo del Estado moderno y la economía de mercado comportó la gradual emergencia de los sistemas públicos de protección social destinados a garantizar, por un lado, el acceso a determinados servicios considerados básicos (típicamente, los servicios sanitarios y educativos) independientemente de la capacidad económica de los individuos (a precios públicos, a título gratuito o como seguro social) y, por otro, la garantía de rentas cuando no se puede vender la mano de obra (prestaciones por enfermedad, desempleo, pensiones y salarios de integración). Es lo que Esping-Andersen (1993) ha denominado la desmercantilización del trabajo y cuyo alcance depende tanto de factores económicos (grado de desarrollo económico), como políticos (grado de organización y capacidad de conflicto de los agentes políticos y tipo de sistema político institucionalizado) e ideológicos (ideologías socio-políticas). La provisión de bienestar a los individuos, sin embargo, no depende sólo del alcance del sistema protección social, sino que es una distribución de responsabilidades entre el estado, el mercado y el denominado tercer sector, integrado principalmente por las familias (junto con las ONGs) (Johnson, 


\section{Gerardo Meil Landwerlin}

1991). En este sentido, el desarrollo del sistema de protección social descansa siempre en unos supuestos sobre cuáles son (e implícitamente deben ser) las responsabilidades de las familias y el mercado en la provisión de bienestar a los individuos.

En las últimas dos décadas una de las preocupaciones centrales sobre el reparto de responsabilidades entre Estado, mercado y familia ha sido el del futuro de la protección social cuestionado por el creciente envejecimiento de la población, entendido éste como crecimiento del número de potenciales pensionistas derivado de una esperanza de vida cada vez más prolongada. El envejecimiento de la población tiene, sin embargo, relación también con la caída de la natalidad, pues los distintos indicadores de envejecimiento tienen como elemento de comparación bien la población total, bien la población jóven, variables afectadas ambas directamente por la natalidad. Y la caída de la natalidad es consecuencia de profundos cambios en la concepción que las nuevas generaciones tienen sobre lo que debe ser la vida familiar, sobre cuáles son las responsabilidades que debe asumir cada uno de sus protagonistas y sobre cómo han de organizarse las relaciones. Este cambio en la concepción sobre cómo debe organizarse la vida familiar plantea numerosos desafíos a la configuración concreta de los sistemas de protección social tal como se han ido construyendo históricamente, pues este cambio está vaciando de contenido varios de los supuestos básicos sobre los que se ha asentado esta construcción y que pueden concretarse resumidamente en la desaparición de la «familia tradicional». En el presente trabajo se abordan las características básicas del cambio familiar para discutir en qué medida suponen un desafío para la operatividad del sistema de protección social y en qué grado se ha adaptado éste a estos desafíos, para finalmente plantear algunas ideas sobre cómo podría producirse esta adaptación. Planteado en términos generales para todos los sistemas de protección social desarrollados, pues en buena medida los desafíos son comunes a todos los países desarrollados, la atención se centrará, no obstante, en el caso español.

\section{La postmodernización de la vida familiar}

La vida familiar en España, como en los demás países desarrollados y como sucede también en otros espacios de la vida social, se encuentra inmersa en un profundo proceso de cambio. Este proceso de cambio afecta a todas las dimensiones de la realidad familiar, dándose tanto en el plano de los modelos ideales de vida familiar, como en el plano 
de los comportamientos efectivos, en la vida familiar diaria. Desde el punto de vista de los modelos ideales de organización de la vida familiar se ha pasado desde el ideal de la familia tradicional entendida como proyecto de convivencia con carácter público para toda la vida y con segregación radical de los roles y de los ámbitos de decisión en función del sexo, a un modelo ideal menos claramente fijado que se distingue, sobre todo, por la pérdida de legitimidad del control social sobre este aspecto de la vida de los individuos y la creciente libertad de conformación individual de los proyectos de convivencia. En este sentido no se trata tanto de la emergencia de un nuevo modelo igualitario, como de la privatización de las opciones sobre cómo se construyen las biografías familiares (Lüscher, 1988; Meil, 1999).

Si el proceso de desarrollo de la familia nuclear moderna responde, como han interpretado los historiadores de la familia, a un proceso de privatización creciente de la vida familiar, a partir de los años sesenta del presente siglo se asiste nuevamente en los países occidentales a un proceso de creciente privatización de la vida cotidiana de los individuos tan radical o más que el que dio lugar a la familia nuclear moderna. Esta nueva privatización ha supuesto el cuestionamiento de la validez de las normas sociales que antaño sujetaban la vida privada de los individuos a un estrecho control social, en favor de una mayor disponibilidad sobre el curso de los comportamientos. $\mathrm{El}$ «qué dirán», que no es sino la manifestación de la interiorización por parte de los individuos de ese control social, es crecientemente sustituido por un «y a ellos que les importa», que marca a un espacio social de libertad individual en la conformación de los proyectos de vida $\mathrm{y}$ en las formas de concebir y organizar la vida en pareja y en familia. En otras palabras, los proyectos y formas de vida familiar se han privatizado y los modelos heredados de organización de la vida familiar han perdido en fuerza vinculante. Las formas de entrada, permanencia y salida de la vida familiar se han flexibilizado quedando las formas que adopte al arbitrio de la negociación y acuerdo entre los protagonistas individuales, o, más precisamente, no siendo legítima la reprobación social de las formas que se apartan de los modelos heredados del pasado. En este sentido, aunque la familia de origen forma parte del proceso de negociación entre los protagonistas, sus márgenes de actuación para condicionar la conformación de los proyectos y formas de vida de sus hijos se han visto fuertemente limitados al haberse erosionado la legitimidad de su intervención. No sólo lo que piensen los vecinos se ha vuelto irrelevante a la hora de decidir en materia familiar, sino que lo que piensen los 
propios padres puede ser puesto igualmente entre paréntesis a la hora de afirmar las opciones individuales.

Esta privatización y la correspondiente reducción del control social ejercido sobre la vida familiar de los individuos han comportado ciertamente una pérdida de importancia del carácter institucional de la familia, esto es, de las formas socialmente establecidas en un momento determinado del tiempo de entender y organizar la vida familiar, pero no así de la importancia y significado subjetivo que para los individuos tiene la vida familiar. A pesar de esta privatización de los proyectos de vida familiar, la vida en pareja no ha dejado de ser el modelo de organización de la vida en común de las personas adultas, pues ni la soltería, ni el vivir sólo, ni el vivir en comunidad, ni lo que podríamos denominar «biografías de pareja flexibles» (Beck, 1986; Beck-Gernsheim, 1998) han emergido como modelos alternativos a la "pareja con vocación de estabilidad», particularmente en el caso español. Ésta, ya sea establecida mediante el vínculo matrimonial o simplemente de hecho, continúa siendo el marco en el cual la gran mayoría de los individuos buscan la satisfacción de sus necesidades afectivas (y sexuales).

Ahora bien, la pérdida del carácter institucional y la correspondiente flexibilización de los modos de organizar la vida familiar han dado lugar lentamente a la emergencia de lo que se ha dado en llamar un «nuevo pacto conyugal» (Roussel, 1989). Este nuevo pacto conyugal se caracterizaría por un mayor énfasis en la legitimidad de las aspiraciones de realización individual de cada uno de los cónyuges, de los yo individuales frente al nosotros pareja e incluso al nosotros familia, cuando existen hijos. Este mayor énfasis en el yo individual frente al nosotros pareja discurre paralelamente a la emergencia de una concepción de la pareja de carácter consensual y más hedonista, más centrada en la satisfacción afectiva y emocional de los dos cónyuges y también más centrada en el ocio conjunto y el consumo mercantil que en la producción doméstica de bienes y servicios. Detrás de la emergencia de este nuevo modelo de comprensión de la vida en pareja se encuentra no sólo la creciente incorporación de la mujer al mercado de trabajo, sino ante todo el profundo cambio en los valores sociales dominantes en dirección hacia un mayor grado de individualización, de búsqueda de espacios de libertad y de voluntad de control de los propios proyectos de vida individual (Beck, 1989).

Corolario de esta nueva concepción de la vida en pareja es también no sólo el reconocimiento del divorcio como fin no biológico a la vida conyugal, sino ante todo del divorcio por mutuo acuerdo cuando los lazos emocionales que fundamentaron la unión han desaparecido. Aun- 
que la presencia de hijos es para una gran parte de la población un importante freno al divorcio, el cambio de valores sobre los que se sustenta el nuevo pacto conyugal también está modificando el papel de los hijos como fundamento de la unión entre los cónyuges, al tiempo que se modifican las relaciones con los hijos.

Paralelamente a este cambio en la concepción de la vida en pareja, el proceso de privatización se ha traducido también en una diferenciación entre sexualidad legítima, matrimonio y procreación, que tradicionalmente constituían dimensiones de una misma realidad denominada familia. Las relaciones sexuales prematrimoniales se han despenalizado y han pasado a formar parte de la normalidad de las relaciones amorosas, al tiempo que el inicio de las relaciones sexuales se produce a edades cada vez más tempranas. A medida que la edad del matrimonio se pospone cada vez más en el tiempo, la edad de inicio de las relaciones sexuales se adelanta cada vez más. Pero al tiempo que las relaciones sexuales prematrimoniales se desestigmatizan y pasan a formar parte de la normalidad del inicio de una biografía amorosa, se refuerza también la exigencia de fidelidad a la pareja, como consecuencia de esa nueva concepción de la pareja. Esta liberalización de las relaciones sexuales ha ido pareja con la emergencia del principio del control de la natalidad y la planificación familiar como normas sociales explícitas. En este sentido, no se trata tanto de la práctica del control de la natalidad, fenómeno que, por otro lado, ya se dio en el pasado durante la transición demográfica, como de la elevación de la planificación familiar al rango de norma social explícita aceptada (y practicada) por la gran mayoría de la población. La norma social que rige las opciones por la procreación establece que sólo se deben tener tantos hijos como se puedan cuidar, educar y atender adecuadamente, esto es, con arreglo a unos estándares cada vez más exigentes. Las relaciones sexuales se han desvinculado así no sólo del matrimonio, sino también de la procreación y ésta tampoco está estrechamente vinculada al matrimonio. Así, la opción por la maternidad en solitario ha comenzado a ser considerada legítima por la mayor parte de la población, lo que no implica que se haya elevado a la categoría de modelo de comportamiento, sino ante todo que ha sido desestigmatizada. Por otro lado, la norma social de que si hay hijos debe haber matrimonio también ha dejado de ser vinculante para la mayor parte de la población. Pero más significativo aún resulta, sin embargo, que el matrimonio ya no implica por sí mismo la procreación, sino que la opción por la no procreación se ha desestigmatizado y la renuncia a la maternidad ya sea por motivos laborales, económicos o de otro tipo ha dejado de estar socialmente estigmatizada. 
Gerardo Meil Landwerlin

TABLA 1: Evolución de los hogares españoles, 1985-1995

\begin{tabular}{||l|r|r|r|r|r||}
\hline \multicolumn{1}{|c|}{ Tipos de hogares } & \multicolumn{1}{c|}{1.985} & \multicolumn{1}{c|}{1.990} & \multicolumn{1}{c|}{1.993} & \multicolumn{1}{c|}{1.995} & $1985 / 1995$ \\
\hline Solo/a de menos de 65 años & 409.670 & 403.136 & 506.750 & 430.779 & $5 \%$ \\
Solo/a de 65 o más años & 496.754 & 720.476 & 882.660 & 870.399 & $75 \%$ \\
Pareja sin niños & 1.582 .029 & 1.876 .636 & 2.060 .977 & 2.015 .167 & $22 \%$ \\
Pareja con 1 niño & 704.541 & 767.919 & 911.346 & 931.536 & $32 \%$ \\
Pareja con 2 niños & 1.142 .825 & 1.067 .021 & 1.081 .604 & 939.195 & $-18 \%$ \\
Pareja con 3 o más niños & 562.854 & 333.421 & 232.533 & 203.641 & $-64 \%$ \\
Adulto con 1 o más niños & 81.259 & 87.730 & 57.682 & 81.923 & $1 \%$ \\
Otro tipo de hogar & 5.490 .749 & 6.093 .643 & 6.156 .521 & 6.459 .694 & $18 \%$ \\
Total & 10.470 .692 & 11.347 .415 & 11.891 .374 & 11.933 .672 & $14 \%$ \\
\hline
\end{tabular}

Fuente: INE (1997), Indicadores sociales de España, Madrid, p. 67, sobre datos de la Encuesta de Presupuestos familiares

Estos profundos cambios en lo que puede denominarse la cultura familiar, esto es, en las representaciones ideales de cómo debe organizarse la vida familiar en sus distintas dimensiones, no se han traducido automáticamente en una crisis de las estructuras familiares, esto es, de cómo viven realmente las familias, pero éstas se están modificando también profundamente, aunque de forma muy distinta de unos países occidentales a otros (Roussel, 1992). En el caso español la desintitucionalización de los proyectos de vida familiar ha sido de menor alcance que en otros países, pero las transformaciones registradas son de profundo alcance (del Campo, 1990; Iglesias de Ussel, 1998; Meil, 1999; Alberdi, 1999). En la tabla 1 se presenta la evolución de distintos tipos de hogares entre 1985 y 1995 y aunque hogares y familias no son términos sinónimos, si nos proporcionan una valiosa información sobre cómo está cambiando la vida familiar en España en la actualidad. Comentaremos brevemente esta tabla y los cambios que subyacen a la evolución que en la misma puede observarse.

En primer término se puede observar cómo el número de hogares de una sola persona encabezados por mayores de 65 años está aumentando de forma rápida, aunque no uniforme. Las razones del rápido crecimiento de este tipo de hogares se encuentra no sólo en la cada vez mayor esperanza de vida de la población española, sino ante todo en la cada vez mayor diferencia de esperanza de vida entre hombres y mujeres, que en la actualidad ya alcanza los 6 años. Pero junto a esta causa demográfica, se encuentra también un cambio en las normas de solidaridad familiar entre las generaciones, de forma que la viudedad 
ha dejado de ser causa para el reagrupamiento de las generaciones. Aunque los contactos y apoyo mutuo entre padres mayores e hijos sigue siendo intenso, la norma que rige la relación de las generaciones es la separación residencial hasta que la edad o la enfermedad hagan inviable este modelo de «intimidad a distancia». De hecho, en un estudio que el autor de estas líneas ha realizado recientemente sobre el cambio en las pautas de solidaridad familiar se concluye en relación a la asunción de responsabilidades de cuidado de los mayores necesitados de ayuda «ciertamente el cuidado por la familia no sólo es la regla, sino también la norma, incluso en una ámbito socioeconómico como el madrileño, fuertemente urbanizado, industrializado y donde el proceso de privatización de los proyectos de vida familiar está ampliamente extendido, pero esta norma está en proceso de redefinición en dirección hacia una solidaridad más «teledirigida», menos absorbente, más de «emergencia». "En este sentido, puede afirmarse que no hay signos claros de una erosión de la solidaridad familiar entre las generaciones, pero «la gran mayoría de los servicios de ayuda mutua que fluyen a través de «la economía solidaria familiar», y que lejos de erosionarse parecen haber aumentado incluso en lo que a los niños se refiere con la postmodernización de la familia, son ayudas limitadas en cuanto al tiempo y/o esfuerzo que requieren para prestarlas, no suponiendo una carga equivalente a la asunción de una segunda jornada laboral. La disposición para prestar este tipo de servicios de ayuda a los familiares próximos no se ha erosionado así con el proceso de privatización registrado en las últimas décadas, ni ha comportado tampoco la exclusión de los flujos de ayuda puntuales a los familiares colaterales (hermanos y hermanas). No obstante, la disposición para asumir servicios de ayuda que impliquen una dedicación temporal prolongada en el tiempo y que requieran un elevado esfuerzo, de forma que constituyan una auténtica carga laboral, creemos que, aunque continúa jugando un importante papel dentro de las redes familiares en la actualidad, está sujeta a un proceso de redefinición en el que se pasa del cuidado exclusivo familiar a dar cabida también a la intervención de cuidadores no familiares, sin que ello implique un desentendimiento por parte de los familiares. Expresado en otros términos, lo que el proceso de privatización ha empezado a cuestionar, creemos, no son las ayudas puntuales más o menos regulares a miembros de la familia, sino fundamentalmente la disposición de los abuelos a asumir el rol de padres cuando sus hijos trabajan (o la de éstos para delegar estas responsabilidades en sus padres) y la disposición (o capacidad) de los hijos (hijas fundamentalmente) a cuidar de sus mayores sin ayuda exterior 
cuando se han vuelto dependientes y necesitan de cuidado continuado» (Meil, 2001b), esto es, el cambio familiar está modificando las posibilidades y predisposiciones a asumir responsabilidades familiares.

Mientras los hogares unipersonales formados por personas mayores está creciendo como consecuencia de estos cambios, los hogares unipersonales encabezados por una persona de menos de 65 años, por el contrario, presentan una evolución muy irregular, aunque con tendencia creciente y ello a pesar del retraso cada vez mayor en la edad de matrimoniar y del aumento de la soltería. La razón de ello hay que buscarla en las dificultades económicas de los jóvenes para vivir de forma independiente, pero también en un profundo rechazo en la cultura española a vivir solo.

Por otro lado, los hogares formados por familias con niños pequeños están disminuyendo de forma continua y acelerada, de suerte que las nuevas familias son cada vez más pequeñas. Detrás de este fenómeno se encuentran dos causas principales. Por un lado, hay que señalar el creciente retraso en la formación de una familia, derivado de la necesidad de los jóvenes de afianzar su posición en el mercado de trabajo, cada vez más inestable, y la necesidad de acumular durante más tiempo el capital necesario para formar un hogar independiente sin que se produzca su desclasamiento. Por otro lado, la caída de la natalidad, sobre la que confluyen multitud de causas pero que están estrechamente relacionados con los cambios culturales arriba indicados, ha reducido el tamaño de la descendencia, al tiempo que se ha ido concentrado cada vez más en el tiempo el período fecundo. Los mayores descensos se han producido en el número de familias numerosas, pero también en el número de familias con dos niños pequeños, al tiempo que el número de parejas con solo un hijo está aumentado de forma continuada.

Al tiempo que ha descendido el número y el tamaño de las familias ha aumentado también el número de parejas sin hijos, aumentando en una década en un $22 \%$. Estas parejas están formadas fundamentalmente por dos tipos muy distintos de configuraciones familiares. Por un lado se trata de parejas de personas mayores que han visto cómo todos sus hijos se han ido emancipando y han formando su propio hogar. Su número está creciendo no sólo por efecto de la prolongación cada vez mayor de la vida, sino también porque han tenido un menor número de hijos que las generaciones precedentes $\mathrm{y}$, por tanto, han necesitado menos tiempo para facilitar a sus hijos la formación de un hogar independiente. Es la fase del ciclo familiar que se ha dado en llamar de «nido vacío», un fenómeno social emergente propio de 
las últimas décadas del siglo XX. También es relativamente nuevo el otro tipo de parejas que conforman este tipo de hogares, pues se trata de parejas que han optado por postponer cada vez más en el tiempo la opción por los hijos y que en muchos casos terminarán por renunciar definitivamente a los mismos, bien porque ya se haya hecho fisiológicamente demasiado tarde, bien por temor a un cambio demasiado drástico en sus estilos de vida. Aunque siempre ha habido parejas que no han tenido hijos, en muchos casos por razones fisiológicas, si bien nunca ha sido un fenómeno muy extendido (Muñoz Pérez, 1993), en la actualidad, como ya se ha indicado, la opción por no tener hijos, al no estar ya socialmente estigmatizada, va extendiéndose cada vez más.

Las familias monoparentales no están ampliamente extendidas y tampoco se evidencia una tendencia claramente creciente, aunque la causa de las mismas sí está modificándose profundamente al tener su origen cada vez más en el divorcio y menos en la muerte. Así, la proporción de familias monoparentales con al menos un hijo menor de 18 años derivadas de la muerte de uno de los padres se ha reducido del 46 al $25 \%$ en la última década, aumentando correspondientemente las derivadas de separación o divorcio (Meil, 2001a). La aceptación generalizada del divorcio por mutuo acuerdo ha alterado profundamente el significado del matrimonio como institución destinada a dar estabilidad a la familia y el sistemático crecimiento que están registrando las rupturas conyugales por separación o divorcio supondrán una extensión de este fenómeno en el futuro. El fuerte empobrecimiento que comportan las rupturas conyugales se traduce en un aumento de los riesgos y los costes derivados la asunción de responsabilidades familiares.

En conjunto podemos afirmar que el cambio familiar se ha traducido en una pluralización de los modos de entrada, permanencia y salida de la vida familiar con una mayor libertad individual para definir el proyecto conyugal y familiar que en el pasado, lo que significa que el modelo tradicional de familia está cada vez menos presente entre las nuevas generaciones, lo que tiene profundas consecuencias para el sistema de protección social.

\section{El modelo de familia subyacente al sistema de protección social}

Los sistemas de protección social pueden organizar la redistribución de la renta y la provisión de servicios públicos con arreglo a dos principios 
básicos claramente diferenciados. Por un lado, el acceso a las prestaciones se puede organizar con arreglo a un principio universalista, en virtud del cual todos los ciudadanos tienen acceso a las prestaciones sociales cuando presentan determinadas características que los hacen acreedores de las mismas (edad para las prestaciones infantiles o para las pensiones de jubilación, edad para el acceso al sistema educativo, quiebra de la salud para el acceso a los servicios sanitarios, etc.). Frente al principio universalista de provisión de bienestar social, esta provisión también puede organizarse con arreglo al principio del seguro social, en virtud del cual las prestaciones sociales se conceden por tener la condición de asegurado o beneficiario, condición que se adquiere mediante el pago durante un período de tiempo determinado (variable según la contingencia de la que se trate) de las cotizaciones sociales legalmente establecidas. En la configuración histórica de los distintos sistemas de bienestar social se ha hablado de una tendencia hacia la convergencia de ambos sistemas, pero aunque en los países que organizan la provisión con arreglo al principio universalista se ha registrado una tendencia hacia la introducción de sistemas complementarios de carácter contributivo y en los países que descansan en un sistema de Seguridad Social de carácter contributivo se han introducido prestaciones de carácter universalista (típicamente los servicios sanitarios y las prestaciones familiares), lo cierto es que las diferencias entre los distintos sistemas siguen siendo de gran alcance, no pudiéndose hablar de convergencia (Esping-Andersen, 1993; Schmidt, 1988; MTSS, 1993).

Mientras los sistemas de protección social de tipo universalista, al considerar al ciudadano como acreedor de la protección social, no descansan en concepción explícita alguna de la familia, lo que no significa que no resulten discriminantes para las mujeres (OConnor, 1996), los sistemas de protección social de tipo contributivo, entre los que se encuentra el español, descansan típicamente en el modelo de familia nuclear tradicional: los individuos contraen matrimonio y forman familias (tienen hijos), el matrimonio dura hasta el final de la vida de los cónyuges y la esposa se encuentra en una relación de dependencia económica directa del marido, centrándose su trabajo en las tareas domésticas, en el cuidado y educación de los hijos y en el cuidado de los miembros de la familia necesitados de ayuda. La protección social se reconoce así al asegurado, esto es, el trabajador que cotiza al sistema de Seguridad Social correspondiente y a través suyo, mediante la técnica de derechos derivados, a todos los familiares dependientes económicamente de él y con los que convive. Los individuos que no quedan 
asegurados a través de esta técnica de protección quedan socialmente desprotegidos. No obstante la tendencia hacia la universalización de la protección que se ha registrado en los países con sistemas de protección social desarrollados ha ido en dirección hacia ampliar los colectivos sujetos a cotización, elevar la edad hasta la que se tiene derecho a prestaciones en calidad de beneficiario y desarrollar sistemas de asistencia social o no contributivos para quienes acrediten estado de necesidad.

Estos supuestos se ven reflejados de distinta forma según los países con este tipo de sistemas de protección social, habiendo además cambiado a lo largo del tiempo a medida que los recursos económicos se han vuelto más escasos, a medida que el principio de no discriminación en razón del sexo ha emergido como un valor básico de la organización social y las legislaciones se han ido adaptando de forma diferencial al cambio familiar. En el caso español, estos supuestos pueden verse muy claramente reflejados tanto en la evolución histórica de las distintas prestaciones reconocidas (familiares, por desempleo, pensiones, etc.) como en las condiciones bajo las cuales se reconocían y aunque sus rasgos sean más nítidamente identificables en la configuración de la protección social en el pasado reciente (véase Iglesias de Ussel y Meil, 2001), también son plenamente identificables en la actualidad.

Un ejemplo muy ilustrativo al respecto lo representa la regulación sobre las pensiones de viudedad. Durante la fase de expansión del sistema de Seguridad Social, en las décadas de los 60 y parte de los 70 , las pensiones de viudedad se reconocían únicamente a las esposas de los asegurados fallecidos, siempre que no volvieran a contraer nuevas nupcias y que no desarrollaran un actividad económica remunerada. Junto con las prestaciones por orfandad aspiraban a sustituir el salario del .marido fallecido. A raíz de la entrada de España en la Unión Europea en 1985 y derivado de la necesidad de trasposición de, entre otras, la directiva de la Unión Europea 79/7/EEC que obliga a la no discriminación por razón de sexo y a la igualdad de trato de hombres y mujeres en los sistemas de Seguridad Social, también los varones pasaron a tener derecho a una pensión de viudedad, al tiempo que se ha suprimido la incompatibilidad con el trabajo remunerado. Aunque la regulación actual de las pensiones ya no está claramente configurada según el modelo de familia tradicional, la existencia de las propias pensiones de viudedad, la imposibilidad de acceder a éstas si no existe vinculo matrimonial previo y su condicionamiento a no contraer nuevo matrimonio así como la existencia de otros muchos derechos derivados de los lazos de parentesco y dependencia económica y residencial del 
asegurado evidencian que, en cualquier caso, la protección social se organiza alrededor de los vínculos familiares. Otro ejemplo de cómo el sistema de protección social descansa implícitamente en el modelo de familia tradicional son los mecanismos públicos para el cuidado de los mayores necesitados de ayuda. Largo tiempo dependientes única y exclusivamente del cuidado de los familiares o de los servicios prestados por el mercado, el desarrollo de los servicios sociales públicos ha estado fuertemente orientado a prestar ayuda a las familias con mayores dependientes (residencias, centros de día, ayuda a domicilio o teleasistencia), aunque a una escala muy limitada en relación a las demandas potenciales, lo que ha redundado en una cobertura pública muy limitada y en la expansión de la oferta privada. Este limitado alcance de la oferta pública evidencia tanto los cambios en la dinámica familiar, como el hecho de que el sistema público partía del supuesto de que este tipo de servicios se prestan en el ámbito familiar, reconociendo la responsabilidad pública para la provisión de este tipo de servicios sólo en los casos definidos explícita o implícitamente como de necesidad (falta de familiares o recursos insuficientes).

La adaptación del sistema de protección social a los cambios familiares arriba indicados ha sido limitada. La aplicación de la directiva 79/7/EEC de la Unión Europea arriba señalada, así como, más en general, el desarrollo de la protección social de la maternidad y la consideración de las dificultades de conciliación de vida familiar y vida laboral como objetivo de la política social son las medidas más destacables en este sentido. Los cambios familiares arriba señalados comportan, sin embargo, desafíos de adaptación de los sistemas de protección social de carácter contributivo de mucho mayor alcance, que en las sucesivas reformas no pueden ser pasados por alto. En lo que sigue trataremos de desentrañar los principales desafíos derivados de las nuevas realidades familiares así como las posibles líneas de actuación.

\section{Desafíos al sistema de protección social derivados del cambio familiar}

Sin ánimo de exhaustividad, en el presente epígrafe vamos a analizar los que consideramos tres principales desafíos, a saber: la caída de la natalidad hasta niveles por debajo del nivel de reemplazo derivada de la libre elección de la opción por la procreación, la incorporación masiva de las mujeres al mercado de trabajo y la creciente importancia de la ruptura familiar derivada de la separación y el divorcio. 
Los desafíos al sistema de protección social...

Desafio derivado de la caída de la natalidad

Como hemos señalado, uno de estos desafíos proviene de la caída de la natalidad y del consiguiente envejecimiento de la población. No es éste contexto para discutir el desafío demográfico al sistema de pensiones derivado del envejecimiento de la población entendido como fuerte crecimiento del número de personas mayores, tan ampliamente discutido en cuanto al alcance del envejecimiento y a la capacidad del sistema de reparto sobre el que se basa la Seguridad Social para hacer frente al volumen de gasto que ello implicará. Lo que se pretende es llamar la atención en este contexto sobre una dimensión de la problemática del envejecimiento y el sistema público de pensiones a la que no se alude en absoluto ${ }^{1}$, pero que, sin embargo, está también en la base del problema. Nos referimos a lo que se ha denominado «cotización demográfica» a la Seguridad Social (vid Meil, 1989) o reconocimiento de la contribución que hacen las familias/mujeres a la viabilidad del sistema de reparto cuando tienen y educan a sus hijos, que en el futuro serán nuevos cotizantes.

$\mathrm{Si}$ en sus albores la Seguridad Social funcionaba sobre la base del principio de capitalización, de forma que las cotizaciones servían, como en las empresas de seguros privadas, para hacer frente al pago de las indemnizaciones o pensiones cuando se materializa el riesgo asegurado, la accidentada evolución que ha conocido a lo largo de su historia, tanto en España como en el resto de países, hizo inviable una financiación de este tipo. Antes o después, como consecuencia de guerras, inflaciones elevadas y persistentes, por la propia universalización de las prestaciones así como por otros motivos, todos los sistemas de Seguridad Social terminaron por financiarse con arreglo al principio de reparto, de forma que los ingresos por cotizaciones de un determinado período de tiempo sirven únicamente para financiar las prestaciones durante ese mismo período de tiempo, dejando eventualmente sólo un margen de maniobra relativamente pequeño de acumulación. En términos macroeconómicos, esto es, como aseguramiento de toda la comunidad nacional, tampoco cabía otra solución (Ventouri, 1954), como se reconoció por la propia comisión encargada de redactar el Pacto de Toledo y que hicieron suyo también los partidos políticos (MTSS, 1995), pues «desde el punto de vista macroeconómico, no existe ninguna acumulación intertemporal de recursos con destino al consumo que, según cual sean las necesidades, pueda en un futuro consumirse pasando a formar parte de la renta nacional; toda acumulación de recursos se convierte en una economía monetaria, en términos macroeconómicos, 


\section{Gerardo Meil Landwerlin}

en capital; el capital ya constituido, sin embargo, no puede reconvertirse en renta nacional, esto es, no puede traducirse en bienes de consumo» ${ }^{2}$. Esto significa, en lo que se refiere a las pensiones, que los recursos necesarios para garantizar la subsistencia física de la tercera edad solamente pueden proceder de los recursos generados por la población cotizante en un momento determinado del tiempo, de ahí que el principio de reparto también se califique como «contrato entre generaciones»o «solidaridad entre las generaciones» (Ventouri, 1954): la generación de adultos, a través de su trabajo, proporcionan a la generación de sus mayores recursos económicos para que puedan vivir económicamente independientes, bajo el supuesto de que cuando ellos sean mayores, la generación de sus hijos harán lo mismo. Pero basar el «contrato de generaciones» solamente en dos generaciones presupone, además de una economía en expansión, la existencia de un crecimiento «natural» de la población que mantenga la relación entre la segunda y tercera generación, esto es, entre activos y pasivos, dentro de unos márgenes razonables. De hecho, la universalización y perfeccionamiento de las prestaciones tras la segunda guerra mundial ha sido posible realizarla ignorando las determinaciones demográficas gracias a una evolución demográfica, además de económica, que ahora se evidencia como excepcional (Kaufmann, 1989). El envejecimiento de la población derivado de una esperanza de vida cada vez mayor ha ido alterando paulatinamente la relación cuantitativa del contrato entre generaciones: a medida que crecía el número de pensionistas, debía crecer la cuantía de ingresos por cotizaciones, salvo que el Estado procediera a una inyección de fondos. Pero el problema no procede sólo de una esperanza de vida cada vez mayor.

Con la caída de la tasa de la natalidad se ha iniciado un proceso paralelo de envejecimiento de la población que amenaza con desequilibrar los fundamentos sobre los que se asienta el sistema de pensiones de la Seguridad Social. Si como consecuencia de la caída de la natalidad se inicia un proceso de reducción del volumen de población, se hace evidente que la amenaza de la operatividad del sistema de reparto o del contrato entre generaciones no procede solamente de la mayor esperanza de vida, y con ello del crecimiento del número de pensionistas, sino que a medida que los efectos de la caída de la natalidad se hacen sentir sobre el volumen de la población activa, los términos en los que se ha «redactado» (por lo poderes públicos) el «contrato entre generaciones» actualmente en vigor y que considera sólo a la generación de adultos y mayores, se hace crecientemente insostenible. No se trata de la «guerra entre generaciones» de la que hablara ya hace tiempo 
Hayek, pero sí, expresado en términos gráficos y exagerados, que la inversión de la pirámide de la población amenaza con hacer saltar por los aires el sistema de la Seguridad, no sólo por su «cabeza», excesivamente pesada, sino ante todo por sus «pies» de barro.

Aunque el número de cotizantes pueda aumentar tanto por la incorporación de las mujeres al mercado de trabajo como en virtud de la inmigración (legal, esto es, que cotiza a la Seguridad Social), de forma que la relación desfavorable entre cotizantes y pensionistas pueda mejorar (supuesto un mercado de trabajo en expansión), a medio y largo plazo es inviable sin tasas de natalidad próximas al nivel de reemplazo generacional. Y además resulta inviable no sólo la protección social, sino la propia supervivencia de la sociedad. Aunque la perentoriedad del problema pueda resultar discutible, lo que sí resulta indiscutible es que la caída del volumen de población producto de la caída de la natalidad, y que tiene sus orígenes en los cambios habidos en el sistema familiar, no son indiferentes para la Seguridad Social. $\mathrm{Y}$ no son indiferentes solamente por sus efectos demográficos, sino ante todo porque el modelo de familia sobre el que se asienta el sistema de pensiones ya no constituye la realidad familiar dominante y cada vez más pierde en vigencia. Con los cambios culturales y familiares a los que se ha hecho referencia en el primer epígrafe, ya no está garantizado que las parejas tengan el número de hijos necesarios para el reemplazo generacional. La desestigmatización de la renuncia a la maternidad, la «indiferencia estructural» en los distintos ámbitos de la sociedad (Kaufmann, 1990) a los beneficios sociales derivados de la tenencia y educación de los hijos, la privatización de los costes de la tenencia de hijos (Iglesias de Ussel y Meil, 2001; Durán, 1999), las legítimas aspiraciones de las mujeres/madres a tener una biografía laboral (y de aseguramiento social) completa, entre otros factores, han elevado sustancialmente los costes directos y de oportunidad de la tenencia de hijos, al tiempo que los beneficios han sido desplazados cada vez más a la esfera inmaterial, lo que no garantiza, visto desde el punto de vista social, el reemplazo generacional. Quienes mantienen la viabilidad demográfica del sistema de reparto, sin embargo, son las mujeres/familias que optan por tener hijos. Por tanto, la Seguridad Social no es sólo un contrato entre dos generaciones, sino entre tres generaciones. Aceptar esta interpretación significa tener que reconocer que las cotizaciones sociales en dinero no recogen todas las contribuciones que se realizan al sistema. Además de las «cotizaciones en dinero", hay «cotizaciones en especie», esto es, hay una «cotización demográfica» que sería necesario tomar en consideración en algún sentido. 
Desafio derivado de la incorporación de la mujer al mercado de trabajo

Estrechamente relacionada con la problemática estobazada hasta el momento está también la creciente extensión de las familias en las que ambos cónyuges trabajan fuera del hogar. La incorporación de las mujeres al mercado de trabajo en principio no representa ningún desafío directo al funcionamiento del principio de reparto, más bien, por el contrario, representa una solución a la creciente reducción de la relación entre cotizantes y beneficiarios derivada del envejecimiento de la población en la medida en que provee de recursos financieros al sistema para hacer frente a los pagos. Por otro lado, el trabajo extradoméstico en la medida en que proporciona recursos propios a las mujeres independientemente de su vinculación conyugal, también reduce, en principio, su vulnerabilidad social y por tanto su potencial dependencia del sistema de protección social. ¿De dónde proceden entonces los desafíos que se plantean al sistema de protección social derivados de la tendencia hacia una incorporación generalizada de las mujeres al mercado de trabajo?. Los desafíos proceden, por un lado, del problema de conciliación de vida familiar y vida laboral y de las consecuencias que de ello se derivan y, por otro lado y estrechamente relacionado con ello, de las desigualdades que se generan según sean las estrategias de las mujeres y de las familias en las distribución de sus capacidades y de su tiempo entre el trabajo remunerado y no remunerado en función de las responsabilidades familiares que asumen.

La organización de la protección social en lo que se refiere a la garantía de rentas está estrechamente asociada al pago de cotizaciones sociales y a la configuración de una biografía de aseguramiento que debe cumplir requisitos cada vez más exigentes en cuanto al período cotizado y a los importes cotizados. La asunción de responsabilidades familiares como la tenencia y educación de niños y el cuidado de los ancianos redunda en el conocido problema de la conciliación de vida familiar y vida laboral, problema que es tanto mayor cuanto mayores sean las responsabilidades familiares a las que hay que hacer frente y que en el modelo de protección basado en la familia tradicional no se producía. La traducción de estos problemas en las biografías laborales $\mathrm{y}$, por tanto, de aseguramiento afecta, dada la actual definición de los roles de genero, casi exclusivamente a la mujeres y difiere sustancialmente de unos casos a otros dependiendo no sólo de las «responsabilidades familiares» a las que hay que hacer frente, sino del 
momento en que éstas aparecen, de la situación del mercado de trabajo, de las aspiraciones profesionales, de la situación económica de la familia, de la confianza del proyecto de vida en común, de las características de la división de tareas entre los cónyuges así como de los recursos disponibles para hacer posible la conciliación, entre otros factores. Según sean las circunstancias, la asunción de responsabilidades familiares puede implicar desde estrés hasta el abandono temporal o definitivo por la mujer del mercado de trabajo. En la medida en que los problemas de conciliación de vida familiar y vida laboral se traducen en distorsiones en la biografía laboral, ya sea por sus consecuencias en la promoción profesional, por la reducción de jornada, por la interrupción temporal o definitiva de la actividad laboral, ello se traduce también en biografías de aseguramiento que redundan en una menor protección individual. En este sentido, y como norma, quienes menores responsabilidades familiares asumen, ceteris paribus, tienen que asumir menores costes en términos laborales y, por tanto, obtienen una mayor renta (además de tener unos menores costes) y, por tanto, dadas las características del sistema contributivo de garantía de rentas, también obtienen una mayor protección social en los casos en los que se materialicen los riesgos sociales protegidos por la Seguridad Social (típicamente mayores pensiones y mayor prestación por desempleo). En la medida en la que el proceso de privatización de los proyectos de vida individual ha erosionado el control social para la asunción de responsabilidades familiares (tenencia de niños y cuidado personal de familiares necesitados de ayuda), la actual configuración de la protección social eleva los costes al tiempo que reduce las ventajas de la asunción de responsabilidades familiares, generando nuevas desigualdades derivadas de las opciones familiares que se toman.

La organización del sistema de distribución y redistribución de la renta (mercado de trabajo, sistema de protección social y sistema de imposición sobre la renta) genera, por tanto, una doble discriminación contra las mujeres/familias que no pueden conciliar vida familiar y vida laboral, generando desigualdades derivadas no sólo de la situación de clase y de género, sino también de las responsabilidades familiares que se asumen. Estas desigualdades se materializan en dos dimensiones distintas, por un lado, en biografías de aseguramiento más irregulares en aquellos casos en los que no se produce un abandono completo del mercado de trabajo y en la ausencia de protección social, así como de las correspondientes rentas del trabajo, en los casos en los que sí se abandona definitivamente el mercado de trabajo. Los casos extremos que ilustran estas desigualdades serían una pareja sin hijos ni mayores 
que cuidar en la que ambos cónyuges trabajan a tiempo completo y una familia numerosa que además cuida de sus mayores necesitados de ayuda que hace imposible el que ambos cónyuges trabajen: mientras en el primer caso la unidad conyugal tras una vida laboral completa habría podido acumular capital para hacer frente a las necesidades de la tercera edad además de obtener dos pensiones de jubilación y una de jubilación y otra de viudedad cuando uno de los dos falleciera (sujetas, no obstante, a un tope máximo), financiadas por las nuevas generaciones de cotizantes, en el segundo caso, las posibilidades de ahorro serían mucho más limitadas (según los casos imposible) y los derechos a garantía de rentas en la tercera edad se limitarían a una pensión de jubilación y a la muerte del esposo a una pensión de viudedad. Todo ello suponiendo en ambos casos que el proyecto de vida en común sólo se rompe con la muerte, porque si éste termina mediante divorcio, las consecuencias económicas diferenciales de ambas estrategias familiares son aún mayores.

¿En qué medida esta reducción de la motivación para la asunción de responsabilidades familiares constituye un desafío para los sistemas de protección social? La problemática puede verse desde distintos puntos de vista. Desde un punto de vista más general puede argumentarse en términos de ausencia real de igualdad de oportunidades entre hombres y mujeres, tal como se argumenta desde la crítica feminista del Estado del bienestar (OConnor, 1996: 50 y ss.; 101 y ss.), afirmando que si bien los derechos sociales estarían garantizados formalmente al no existir barreras formales de género a la incorporación al mercado de trabajo y a los derechos a que dan lugar las cotizaciones sociales, en la práctica, sin embargo, ello se traduce, como hemos visto, y dada la definición actual de los roles de género, en oportunidades muy diferentes tanto en el mercado de trabajo como reproducidas posteriormente por el sistema de protección social.

Desde un punto de vista más próximo a la lógica de la organización de la protección social sobre la base del principio contributivo, los desafíos se presentan en distintas dimensiones. Por una parte se encuentra la ya referida disminución de la motivación para la procreación y su amenaza a medio plazo para la operatividad del principio de reparto. Por otra parte se encuentra la disminución de la motivación para el cuidado y atención de familiares dependientes y en particular de los mayores, teniendo en cuenta el proceso de acelerado envejecimiento de la población y el consiguiente aumento de personas mayores necesitadas de ayuda. En la medida en la que el sistema de protección social no ha sido ajeno a las necesidades de cuidado de los mayores, 
desarrollando servicios de cuidado personalizado con distintas características (residencias, centros de día, servicios de respiro y atención a domicilio), mayormente sobre la base del principio de subsidiariedad (cobertura allí donde no llega el cuidado familiar o el mercado), la reducción de la motivación y de las posibilidades de los hijos para hacer frente al cuidado de sus mayores, a la luz de la implicación de ambos cónyuges en el mercado de trabajo, plantea un reto al sistema de protección social. Las respuestas a este reto pueden ser muy liberales, haciendo recaer exclusivamente en las familias y en el mercado las responsabilidades de la provisión y financiación de servicios de cuidado, o más intervencionistas contribuyendo directamente a hacer frente a estas necesidades a través de la provisión de servicios o indirectamente a través de la financiación total o parcial de servicios de cuidado ofertados por el mercado. En la medida en que la respuesta no sea liberal, la ausencia de reconocimiento de alguna forma de los beneficios sociales derivados del cuidado de los mayores en el contexto familiar, se traducirá en una mayor demanda de recursos públicos para el cuidado de los mayores necesitados de ayuda, tanto más cuanto más vaya desestigmatizándose el cuidado por otras personas que no sean familiares, lo que redundará en una mayor desigualdad y discriminación contra las mujeres/familias que asumen estas responsabilidades familiares.

Por otra parte, la creciente incorporación de las mujeres al mercado de trabajo de forma permanente va a poner en cuestión la legitimidad de las pensiones de viudedad. Introducidas por primera vez en 1911 en Alemania (Venturi, 1954) y concebidas para hacer frente a las dificultades económicas derivadas de la muerte del sustentador principal de la unidad familiar (siempre que la mujer no pudiera trabajar para contribuir al sostenimiento de la familia, por tanto, concebidas a partir de un modelo de organización familiar de tipo tradicional), su razón de ser no deriva de la transmisión a los herederos de un derecho de propiedad sobre unos capitales (cotizaciones) aportados durante la vida laboral, sino como compensación por la pérdida de ingresos derivadas ıa muerte del cónyuge, supuestamente, al menos, durante la fase de expansión del sistema, el sustentador principal de la unidad familiar. En realidad podría decirse que la pensión de viudedad era (hasta la extensión del derecho a la misma también a los varones) la institucionalización modernizada del "precio de la novia». La razón de ser de la pensión de viudedad, como hemos visto más arriba, deriva así de un modelo de familia que estando plenamente vigente entre las generaciones más mayores, entre las generaciones más jóvenes es cada vez más extraño. 


\section{El desafío derivado del divorcio}

El tercer desafío para el sistema de protección social derivado del cambio familiar es la emergencia de la ruptura conyugal por separación o divorcio. Fenómeno apenas extendido en el pasado, aunque tampoco inexistente, al menos, en la forma de abandono del hogar (el denominado «divorcio de los pobres»), es en la actualidad cada vez más frecuente y además socialmente cada vez más aceptado, de forma que, aunque las parejas no inicien su proyecto de vida en común pensando en que terminará en una separación, ésta ha pasado a formar parte del horizonte de expectativas posibles a considerar. Al hilo del cambio familiar arriba apuntado, el matrimonio ha dejado así de ser un vínculo para toda la vida, transformando profundamente la componente de aseguramiento de satisfacción de necesidades vitales que tenía en el pasado y ello, aunque de distinta forma, tanto para el hombre (cuidado personal) como para la mujer (garantía de ingresos). La emergencia de la posibilidad del divorcio en el horizonte del proyecto de vida en común altera, por tanto, profundamente las condiciones del tradicional contrato de matrimonio, haciendo que los costes de asunción de responsabilidades familiares y, en consecuencia, de dedicación al trabajo no remunerado (y tampoco socialmente protegido) se eleven sustancialmente. En este sentido, el problema de la conciliación de vida familiar y vida laboral cobra una nueva dimensión de riesgo, sobre todo, para las mujeres, máxime cuando la protección social que se reconoce para los casos de ruptura conyugal y las correspondientes dificultades económicas que de ello se derivan son compensadas únicamente para los casos de ruptura por muerte de uno de los cónyuges (pensiones de viudedad y pensiones de orfandad). En la actualidad, sin embargo, el divorcio ha sustituido a la muerte como principal causa de monoparentalidad y afecta cada vez más a las mujeres, incluso en un país como España donde las tasas de divorcialidad son relativamente bajas. Como consecuencia de las dificultades económicas generadas por la ruptura, y al no existir protección social alguna, junto con la escasa compensación pública de cargas familiares, el divorcio, particularmente cuando se han asumido responsabilidades familiares, ha pasado a ser una nueva fuente de pobreza. De hecho en casi todos los países de la Unión Europea (significativamente a excepción de los nórdicos) los hogares monoparentales se encuentran sobrerepresentados entre los hogares más pobres (Eurostat, 2000). En este sentido son numerosos los autores que se refieren al divorcio como un «nuevo riesgo social», incluso el propio Consejo de Europa (MTSS, 1989), riesgo social al que apenas 
Los desafíos al sistema de protección social...

se han adaptado los sistemas de protección social. No obstante, al respecto es preciso señalar que no es tanto el divorcio como tal el que emerge como un «nuevo riesgo social», sino la existencia del divorcio cuando se han asumido cargas familiares y singularmente con la presencia de hijos.

\section{Alcance y limitaciones de la adaptación del sistema de protección social a los desafíos derivados del cambio familiar}

¿En qué medida se han adaptado los sistemas de protección social a los desafíos derivados del cambio familiar? En general, hay una amplia evidencia de que los sistemas protección social de tipo socialdemocrático (universalistas) se han adaptado mejor a estos cambios, mientras que otros sistemas de protección social lo han hecho de forma mucho más limitada (OConnor, 1996; Esping-Andersen, 2000). No obstante, impulsado por la emergencia del principio de no discriminación por razón de sexo y de la garantía de la igualdad de oportunidades de hombres y mujeres como valores centrales de la organización social, todos los sistemas de protección social desarrollados, aunque de forma diferencial han desarrollado políticas más o menos coherentes tendentes a promover la conciliación de vida familiar y vida laboral (OCDE, 1995), impulsadas en buena medida por instituciones supranacionales (OIT, Comisión de la Unión Europea). En algunos casos, estas políticas se han articulado con las políticas familiares destinadas a compensar socialmente los costes derivados de la tenencia de los hijos, aunque tendiendo a centrar esta compensación mayormente en las familias con mayores cargas (ya sea por el número de hijos, por la edad de los mismos o por el nivel de renta de la unidad familiar). En el caso español (y en general en todos los países del sur de Europa (Flaquer, 2000)) las políticas de conciliación han pasado, sin embargo, a ser el eje central de la política familiar al tiempo que la dimensión de la política de compensación pública de responsabilidades familiares ha desaparecido mayormente como objetivo explícito del sistema de redistribución de la renta (Iglesias de Ussel y Meil, 2001).

La política de conciliación de vida familiar y vida laboral en España se ha ido desarrollando lentamente, como ha sucedido, por otro lado, también en los demás países de la OCDE, y ha estado presidida, sobre todo, por la facilitación de la permanencia en el empleo de las madres, más que por la promoción de la salida temporal del mercado de trabajo 
para posibilitar la plena dedicación al cuidado de los niños, compensando los costes derivados de esta opción. Esta facilitación de la permanencia en el empleo se ha promovido, por un lado, a través del adelantamiento de la entrada en el sistema escolar hasta los 3 años (política que no obstante no ha estado definida tanto por la problemática de la conciliación como por criterios específicos de política educativa) y por otro por una política de facilitación de permisos laborales cada vez más prolongados y más seguros. Los costes derivados de estas opciones se han hecho recaer fundamentalmente en los/as posibles beneficiarios/as siendo compensados sólo de una forma muy limitada, lo que ha redundado, dadas también la precariedad en el empleo y el elevado desempleo existente, en un recurso muy limitado a los permisos. Esta circunstancia junto con el escaso alcance de la compensación pública de cargas familiares a través de las prestaciones familiares de la Seguridad Social y la tributación de la renta ha hecho que los costes directos e indirectos derivados de la aunción de responsabilidades familiares sólo hayan sido compensados de forma muy limitada, lo que evidencia que la adaptación a los desafíos arriba indicados ha sido más bien escasa. No obstante, se han producido avances que es preciso desarrollar de forma algo más pormenorizada.

Las primeras medidas que han ido más allá del permiso de maternidad y lactancia datan de la introducción del Estatuto de los trabajadores en 1980 y los desarrollos posteriores han venido en buena medida determinados, y casi se podría decir incluso impuestos, por la necesidad de trasposición de las directivas comunitarias en esta materia a la legislación española. El Estatuto de los Trabajadores y la Ley 8/1980 introdujeron de una forma muy restrictiva los principales mecanismos que se han arbitrado para promover la conciliación. Así, se amplió el permiso de maternidad de 12 a 14 semanas, se estableció la posibilidad legal de pedir reducción de jornada laboral de $1 / 3$ o la $1 / 2$ con la correspondiente reducción de salario, se estableció el derecho a la reducción de hasta 1 hora por lactancia y se estableció la excedencia por maternidad, pero sin garantía alguna de reincorporación.

La legislación posterior, que data de 1989 (Ley 3/1989), 1995 (Ley 4/1995) y 1999 (Ley 39/1999), se ha centrado en los siguientes aspectos:

- Ampliar la cobertura, al incluir en 1989 los hijos adoptados y en 1999 el cuidado de familiares dependientes y extender el permiso de maternidad hasta las 16 semanas y el de excedencia hasta los 3 años. 
- Al mismo tiempo se ha introducido la posibilidad de que los padres varones disfruten también de hasta cuatro semanas del permiso de maternidad así como tengan derecho a pedir la excedencia por maternidad, aunque no se ha llegado todavía a configurar un derecho de paternidad específico, esto es, destinado sólo a los padres varones para promover una distribución más igualitarias de las responsabilidades familiares entre ambos cónyuges.

- Aumentar las garantías para la vuelta al trabajo al establecer el derecho a reserva del puesto de trabajo durante el primer año de excedencia y del vínculo laboral hasta los 3 años y a partir de 1999 al prohibir el despido por causa de maternidad.

- Reducir parcialmente los costes derivados de los permisos tanto para el trabajador como para el empresario al configurar en 1995 el seguro de maternidad como una contingencia específica con derecho a un salario de sustitución del $100 \%$ en lugar del $75 \%$ de la Incapacidad Laboral Transitoria, al computar la excedencia del primer año como antigüedad a todos los efectos y como cotizado a efectos de la biografía de aseguramiento a la hora de computar períodos de carencia o de cotización para el acceso a las prestaciones de la Seguridad Social y al exonerar a las empresas de las cotizaciones sociales de los contratos de sustitución (en régimen de interinidad) de las trabajadoras/es que sean beneficiarios de la excedencia por cuidado de menores a cargo.

- La reducción de salario derivada de la reducción de jornada o su pérdida por la utilización de la excedencia no encuentran compensación alguna, ni de forma directa a través de la Seguridad Social, ni indirecta a través de los impuestos (salvo la lógica reducción del tipo impositivo aplicable por menor renta y la deducción por mínimo vital), como sí sucede, sin embargo, aunque con restricciones, en otros países como Dinamarca o Alemania.

La ley 39/1999 ha introducido la importante novedad de contemplar también la posibilidad de la utilización de algunos de estos instrumentos para el cuidado de familiares de hasta segundo grado que por razones de edad o enfermedad no puedan valerse por sí mismos. En concreto se han introducido los siguientes permisos:

- Dos días retribuidos por enfermedad grave, accidente, hospitalización y fallecimiento de parientes hasta $2^{\circ}$ grado. 
- Posibilidad de acogerse también a la reducción de entre 1/3 y 1/2 la jornada laboral, con disminución proporcional del salario y de los derechos sociales que de ello se derivan.

- Excedencia en los mismos términos que por cuidado de niños, pero por máximo de 1 año y no siendo de aplicación ni la bonificación del 100 por 100 de la cuota empresarial a los contratos de interinidad que se celebren con desempleados, ni el cómputo a efectos de carrera de aseguramiento del tiempo que dure el permiso.

Para valorar adecuadamente el alcance de esta adaptación al desafío derivado del cambio familiar es preciso dirigir la atención aunque sea brevemente a las características de la compensación pública de cargas familiares a través de los mecanismos de redistribución de la renta (prestaciones de la Seguridad Social y tratamiento fiscal de la renta), a fin de evidenciar, como se ha afirmado más arriba, cómo los costes derivados de la asunción de responsabilidades están mayormente privatizados. A partir de 1990 las devenidas ridículas (por su cuantía) prestaciones familiares de la Seguridad Social se refundieron en una sola prestación por hijo a cargo destinadas a las familias con menores ingresos o con hijos minusválidos físicos, psíquicos o sensoriales y ello independientemente de su condición de asegurados o no, convirtiéndose así en una prestación destinada fundamentalmente a luchar contra la pobreza infantil, además de proteger socialmente a las familias con hijos minusválidos. Aunque de cuantía sustancialmente superior a las anteriores prestaciones por hijo a cargo, su importe (36.000 pts. anuales hasta el año 2000, año en que se actualiza su valor en relación a la inflación hasta situarlas en 48.420 pts. anuales) apenas supone mejora alguna en la renta de las familias (salvo que exista un elevado grado de minusvalía) y ello a pesar de que se trata de familias de renta baja, no contribuyendo, por tanto, prácticamente en nada a reducir la pobreza infantil. El reconocimiento que se hace de los costes derivados de la presencia de hijos a cargo en la tributación sobre la renta tampoco redundan en una mejora sustancial de la renta. Aunque la nueva regulación del IRPF ha significado un aumento no despreciable de la renta disponible neta después de impuestos cuando hay hijos a cargo respecto a la regulación anterior a 1999, la mejora, como puede comprobarse en la tabla 2, apenas si representa compensación alguna ${ }^{3}$. 
Los desafíos al sistema de protección social...

TABLA 2: Renta disponible neta de impuestos y transferencias familiares como porcentaje de la renta bruta deducidas las cotizaciones sociales según distintos niveles de renta y situaciones familiares (renta 1999)

\begin{tabular}{|l|c|c|c|c||}
\hline & Solo & $\begin{array}{c}\text { Matrimonio } \\
\text { sin hijos }\end{array}$ & $\begin{array}{c}\text { Matrimonio } \\
\text { con dos } \\
\text { hijos }\end{array}$ & $\begin{array}{c}\text { Matrimonio } \\
\text { con tres } \\
\text { hijos }\end{array}$ \\
\hline 1 ingreso de 0,5 el salario medio & 100 & 100 & 100.62 & 108.38 \\
1 ingreso igual al salario medio & 86 & 91.12 & 95.31 & 97.70 \\
1 ingreso de 1,5 el salario medio & 81.73 & 85.76 & 88.77 & 90.89 \\
1 ingreso de 1,66 el salario medio & 80.77 & 84.40 & 87.38 & 89.46 \\
2 ingresos, uno igual al salario medio, & & & & \\
el otro 0,66 el salario medio & 80.77 & 88.38 & 90.75 & 92.34 \\
1 ingreso igual 2 veces el salario medio & 78.47 & 82.24 & 84.91 & 86.49 \\
2 ingresos, ambos igual al salario medio & 78.47 & 86 & 88.10 & 89.61 \\
\hline
\end{tabular}

Leyenda: El valor 100 significa que se recibe el $100 \%$ de los ingresos brutos y, en su caso, prestaciones por hijo a cargo de la Seguridad Social. No se incluye en el cómputo los supuestos de presencia de minusvalías.

Fuente: Adaptado de Iglesias de Ussel y Meil, 2001, p. 234

A pesar de las importantes mejoras en la protección social, la adaptación a los desafíos planteados por el cambio familiar presenta significativas limitaciones, pues apenas reconoce la rentabilidad social de la asunción de responsabilidades familiares no compensando prácticamente nada los costes directos derivados de dicha asunción y esta adaptación, al estar fundamentalmente centrada en facilitar la conciliación de vida familiar y vida laboral, tiende a perjudicar a aquellas familias organizadas con arreglo al modelo tradicional. En este sentido, por tanto, los costes derivados de la asunción de responsabilidades familiares están mayormente privatizados y además, en realidad, doblemente privatizados pues los costes en los que las familias incurren para hacer frente a estas responsabilidades están sujetos a tributación sobre la renta (además del correspondiente IVA). Así, los costes de los sistemas de cuiado de niños o mayores (guardería, asistenta u otros que impliquen un pago), a pesar de resultar imprescindibles para obtener la segunda renta o para posibilitar la conciliación no son considerados gastos deducibles. En este sentido, lejos de reforzar la motivación para la asunción de responsabilidades familiares, la adaptación del sistema de protección social a los desafíos derivados del cambio familiar tiende a la desmotivación, no tanto porque no promueva la conciliación de vida familiar y vida laboral, como por el hecho de no considerar los costes que para las familias tienen. 
En efecto, los costes directos de la tenencia de los hijos se hacen recaer prácticamente en exclusividad sobre las familias y la opción por la tenencia de un número mayor de hijos que compense la tendencia a la minimización del tamaño de las familias carece a efectos prácticos de reconocimiento alguno. El hecho de que la política de permisos laborales haga recaer también los costes directos del recurso a los mismos en las propias familias (los permisos pueden ser utilizados tanto por los hombres como por las mujeres) se encuentra dentro de la misma filosofía de consideración de las opciones a favor de la asunción de responsabilidades familiares como una decisión privada y dado que los permisos son utilizados casi exclusivamente por las mujeres, resulta discriminatorio para éstas desde el momento en el que las cotizaciones a la Seguridad Social se hacen a título individual y no la de ambos cónyuges conjuntamente. En términos de su reconocimiento a efectos de biografía de aseguramiento, se ha producido una mejora al reconocerse como cotizado hasta un año del permiso por cuidado de menores a cargo, lo que supone un paso, aunque tímido, hacia el reconocimiento de la "cotización demográfica», que de reconocerse, debería aplicarse a todas las familias o mujeres que tuvieran hijos, independientemente de su relación laboral y del recurso o no a los permisos. No obstante, si los permisos pueden extenderse hasta tres años no se entiende que el reconocimiento como cotizado se limite a un año máximo y no a toda la duración de la excedencia.

La adaptación del sistema de protección social a la extensión del divorcio apenas si se ha dado. La principal mención que se hace al respecto en la Ley de Seguridad Social es la que establece que el derecho a la pensión de viudedad, en los casos de separación o divorcio, corresponderá a quien sea o haya sido cónyuge legítimo y en cuantía proporcional al tiempo vivido con el cónyuge fallecido (art. 174.2). Respecto al derecho a una pensión de jubilación, ésta sólo se reconoce al asegurado una vez cumplidos los requisitos legalmente establecidos, pero no al excónyuge en función de la duración del matrimonio (salvo que tenga derecho a una pensión de jubilación propia), por lo que las desigualdades que se generan en la familia en función del género en relación a la distribución del tiempo entre el trabajo remunerado y no remunerado se reproduce por el sistema de protección social (salvo en la garantía de la pensión de viudedad) y sólo queda recogido en la obligación de la prestación de alimentos fijada por el juez en el convenio regulador de la separación, una protección que resulta muy débil. Más lógico, al menos cuando el régimen económico del matrimonio es de gananciales, sería que las cotizaciones a la Seguridad Social 
fuesen consideradas comunes y se computaran dividiendo las aportaciones como derechos diferenciales de cada cónyuge al sistema, aunque con la configuración actual del sistema en la que se tiene en cuenta el total de años cotizados pero sólo las bases reguladoras de los últimos quince años eso supone reconocer dos veces la cotización, lo que discriminaría a su vez a las mujeres casadas que cotizan a la Seguridad Social, pues pondría en un mismo plano a quienes cotizan en dinero y eventualmente en responsabilidades familiares con quienes sólo cotizan con responsabilidades familiares.

La privatización de los proyectos de vida familiar que está teniendo lugar en la actualidad no tiene por qué traducirse en una privatización de los costes de la asunción de responsabilidades familires. La asunción de responsabilidades familiares tiene beneficios sociales que es preciso reconocer, máxime cuando cada vez está menos garantizada dicha asunción por parte de las nuevas generaciones y cuando la percepción de los costes derivados de dichas opciones se hacen más evidentes. Argumentando desde el punto de vista económico, puede afirmarse que desde el punto de vista social es indiferente que las familias elijan entre vacaciones o la compra de un coche, pero en absoluto es indiferente que elijan entre asumir responsabilidades familiares (tener un hijo, cuidar de un mayor necesitado de ayuda), comprar un coche o ir de vacaciones. Aunque los costes económicos, en dinero, no son ni mucho menos la única, ni la principal motivación para la asunción de responsabilidades familiares, las familias a la hora de elaborar sus estrategias no son indiferentes a las restricciones económicas. Por ello el sistema de protección social debería responder de una forma más perceptible para la ciudadanía a los desafíos del cambio familiar.

\section{Algunas consideraciones sobre cómo podría adaptarse el sistema español de protección social al desafío del cambio familiar}

¿Cómo podría responder el sistema de protección social español a los desafíos derivados del cambio familiar? La respuesta a esta pregunta es necesariamente política, en el sentido de que debe reflejar unos valores determinados y, por tanto, una visión sobre lo que es deseable y no es deseable. En las posiciones políticas en relación a la configuración de la protección social española durante las pasadas décadas ha habido una, desde nuestro punto de vista, falsa contraposición entre la promoción de la incorporación de la mujer al mercado de trabajo y la 
protección social de la familia, como si ambas dimensiones fueran mutuamente excluyentes. Partiendo desde una postura pluralista, en la que se trata de respetar las opciones individuales, la adaptación de la protección social a los cambios familiares debería suponer el reconocimiento de la rentabilidad social derivada de la asunción de responsabilidades familiares y los costes directos y de oportunidad, con riesgo de pobreza incluido, que las mismas tienen para las familias que las asumen, pero sobre todo, dada la actual división del trabajo entre los géneros, de su coste para las mujeres. La adaptación de la potrección social debería, por tanto, garantizar la libertad de opción al tiempo que no penaliza doblemente a quienes optan por asumir las responsabilidades familiares. Por tanto, no se trata de que el Estado asuma las responsabilidades familiares (importación de trabajadores y su integración, desarrollo de servicios sociales, etc.), sino que las tome en consideración a la hora de la redistribución de la renta.

Sin ánimo de exclusividad, algunas medidas que podrían considerarse en este sentido podrían ser las siguientes:

1. Introducción de una "cotización demográfica», que podría consistir en el reconocimiento como cotizados de al menos tres años por cada hijo habido, pudiendo eventualmente cualificarse en función del status de familia numerosa, pero en cualquier caso independientemente del status laboral. Dada la actual discriminación en razón del sexo (y del status maternal) este reconocimiento debería computarse en las biografías de aseguramiento de las mujeres.

2. La consideración de las cotizaciones a la Seguridad Social como parte de los bienes gananciales y su reparto entre los cónyuges como parte del patrimonio de reparto en caso de ruptura, de forma que dieran lugar a derechos propios para cada uno de los cónyuges. En este sentido, el tiempo cotizado podría dividirse entre dos, introduciendo la posibilidad de cotizaciones adicionales a fin de compensar la reducción del número de años cotizados y no introducir la discriminación a la que se ha aludido anteriormente. Una consecuencia de esta medida sería elevar aún más los costes del divorcio, sobre todo, para el hombre, que así estaría más interesado y, quizá, apoyaría más activamente el trabajo extradoméstico de su mujer.

3. La equiparación de viudedad y separación carece de pertinencia, tanto por motivos doctrinales como por el enorme incentivo al fraude que incluiría, pero una extensión de las pensiones de 
orfandad sujetas a prueba de necesidad en los casos de separación no carecería de fundamento. No obstante, tiene mucho más sentido, la fijación de las prestaciones por hijo a cargo (sujetas de hecho a prueba de ingresos) en unos niveles que reduzcan de forma efectiva los riesgos de pobreza infantil, garantizando un mínimo existencial (como se supone en el IRPF) a todos los menores (independientemente del estado civil de sus padres).

4. Más problemático resulta el reconocimiento del cuidado de familiares necesitados de un cuidado intensivo en tiempo. En este caso, el seguro de cuidado en la vejez introducido en Alemania podría ser un punto de partida para la discusión.

La privatización de los proyectos de vida familiar que está teniendo lugar en la actualidad no tiene por qué traducirse en una privatización de los costes de la asunción de responsabilidades familires. La asunción de responsabilidades familiares tiene beneficios sociales que es preciso reconocer, máxime cuando cada vez está menos garantizada socialmente dicha asunción por parte de las nuevas generaciones y cuando la percepción de los costes derivados de dichas opciones se hacen más evidentes y perceptibles para los propios implicados. Argumentando desde el punto de vista económico, puede afirmarse que desde el punto de vista social es indiferente que las familias elijan entre vacaciones o la compra de un coche, pero en absoluto es indiferente que elijan entre asumir responsabilidades familiares (tener un hijo, cuidar de un mayor necesitado de ayuda), comprar un coche o ir de vacaciones. Aunque los costes económicos, en dinero, no son ni mucho menos la única, ni la principal motivación para la asunción de responsabilidades familiares, las familias a la hora de elaborar sus estrategias no son indiferentes a las restricciones económicas. Por ello, el sistema de protección social debería responder de una forma más perceptible para la ciudadanía a los desafíos del cambio familiar.

\title{
Referencias bibliográficas
}

\author{
Alberdi, I. (1999), La nueva familia española, Taurus, Madrid. \\ BECK, U. (1986), Risikogesellschaft, Suhrkamp, Frankfurt am Main. \\ Beck-Gernsherm, E. (1998): Was kommt nach der Familie? Einblicke in neue Lebens- \\ formen, Beck Verlag, München. \\ CAmpo, S. del (1991), La nueva familia española, Eudema, Madrid.
}




\section{Gerardo Meil Landwerlin}

CAmpo, S. del (1995), "La política familiar en el franquismo» y "La política familiar en la democracia» en Familias: Sociología y Política, Universidad Complutense, Madrid.

CONSEJO DE EUROPA (1989), «Informe sobre la Seguridad Social en una sociedad cambiante» en Ministerio de Trabajo y Seguridad Social (1992), La Seguridad Social en una sociedad cambiante, Madrid, colección Seguridad Social.

Duran, $M^{a}$ Angeles (1999), "Tendencias en el coste de la parentalidad en Europa" en Revista del Ministerio de Trabajo y Asuntos Sociales. Asuntos Sociales, nro. extraordinario sobre la conciliación de la vida familiar y vida laboral.

Eurostat (2000), Income poverty and social exclusion in the European Union, Luxemburgo.

EsPing-ANDERSEN, G. (1993), Los tres mundos del Estado de bienestar, Alfons el Magnanim, Valencia.

Esping-Andersen, G. (2000), Fundamentos sociales de las economías postindustriales, Ariel, Barcelona.

FlaqUer, Ll. (2000), "Is there a southern european model of family policy» n A. Pfenning y Th. Bahle (eds), Families and Family Policies in Europe, Peter Lang Verlag, Frankfurt.

IgLesias de Ussel, J. y MEIL, G. (2001), La política familiar en España, Ariel, Barcelona

JOHNSON, N. (1991), El Estado de bienestar en transición, Ministerio de Trabajo y Seguridad Social, Madrid-

KAUfMANN, F.X. (1988), "Sozialpolitik und Bevölkerungsprozess» en HECK, B. (ed), Sterben wir aus. Die Bevölkerungsentwicklung in der BRD, Freiburg a. Br.

Kaufma.n, F.X. (1990), Zukunft der Familie, Beck Verlag, München.

Luскничs, L. y WARD, S. (1997), “Equal pension rights for men and women: a realistic perspective?» en Bosco, A. y Hutsebaut, M. (eds), Social protection in Europe, European Trade Union Institute, Bruselas.

LÜscheR, K.; Schultheis, F.; Wehrspaun, M. (eds) (1988), Die postmoderne Familie, Universidad Konstanz, Konstanz.

Ministerio de Trabajo y Seguridad Social (1993), Convergencia de políticas sociales, colección Seguridad Social, Madrid.

Minsterio de Trabajo y Seguridad Social (1995), Pacto de Toledo, colección Seguridad Social, Madrid.

Ministerio de Trabajo y Seguridad Social (1997), La mujer y la protección social, colección Seguridad Social, Madrid.

MeIL, G. (1.989), "Seguridad Social y familia" en Revista de la Seguridad Social, 42, $1.989,43-56$.

MeIL, G. (1999), La postmodernización de la familia española, editorial Acento, Madrid.

MeIL, G. (2001a), "Aspectos demográficos de la familia en España» en Revista de Educación (en prensa).

MEIL, G. (2001b), «Hogares nucleares y familias plurigeneracionales» en VARIOS AUTORES, Estructura y Cambio Social, libro homenaje a S. del Campo, Centro de Investigaciones Sociológicas, Madrid.

Muñoz Pérez, F (1995): «Las parejas sin hijos en Portugal y España» en Revista Española de Investigaciones Sociológicas, 70, pp. 39-66.

Oconnor, J. (1996), «From Women in the Welfare State to Gendering Welfare State Regimes» en Current Sociology, 44, 2. 


\section{Los desafíos al sistema de protección social...}

CDE (1995): «Long-term leave for parents in OECD countries» en Employment Outlook, july 1995, Paris, pp. 171-200.

Rousses, L. (1.992), “La famille en Europe Occidentale: Convergence et divergences» en Population, 1 (traducido en Infancia y sociedad, 16, pp. 103 a 120).

Schмidт, M. ( 1988), Sozialpolitik, Lesk+Budrich, Opladen.

Venturi, A. (1954), Los fundamentos científicos de la Seguridad Social, Ministerio de Trabajo y Seguridad Social, colección Seguridad Social, Madrid 1994.

\section{Notas}

* Este trabajo ha sido financiado parcialmente por el Ministerio de Educación y Ciencia, DGCYT, Proyecto PB97-0046, a quien el autor agradece el apoyo recibido.

** Profesor Titular de Sociología de la Universidad Autónoma de Madrid, gerardo.meil@uam.es.

1 Véase, por ejemplo, el texto del Pacto de Toledo (MTAS, 1995), Herce, J.A. y Alonso Meseguer, J. (2000), La reforma de las pensiones ante la revisión del Pacto de Toledo, Servicio de estudios de la Caixa, Barcelona; Carpio, M. (dir) (2000), El sistema de pensiones, una reforma sin fin, Price Waterhouse Coopers, Madrid; Blanco, F. (1999), Redistribución y equidad en el sistema español de pensiones de jubilación. Las reformas del Pacto de Toledo, Consejo Económico y Social, Madrid.

2 Mackenroth en Döring, D. (1980), Das System der gesetzlichen Rentenversicherung, Frankfurt a. M., p. 125.

3 Para más detalles sobre el alcance de la compensación pública de cargas familiares, tanto desde el punto de vista de su peso en el sistema de protección social como de su impacto en la renta disponible de los distintos tipos de familias y su capacidad para hacer frente a los riesgos derivados de la asunción de responsabilidades familiares véase Iglesias de Ussel y Meil, 2001, capítulo 4. 